EUROPEAN JOURNAL OF PURE AND APPLIED MATHEMATICS

Vol. 14, No. 4, 2021, 1507-1516

ISSN 1307-5543 - ejpam.com

Published by New York Business Global

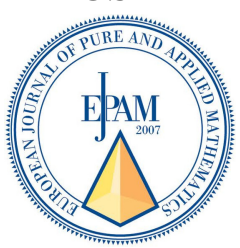

\title{
On Micro-generalized closed sets and Micro-generalized continuity in Micro Topological spaces
}

\author{
Taha H. Jasim ${ }^{1}$, Saja S. Mohsen ${ }^{2, *}$, Kanayo S. Eke ${ }^{3}$ \\ ${ }^{1}$ Department of Mathematics, college of computer Science and Mathematics, \\ Tikrit University, Tikrit, Iraq. \\ ${ }^{2}$ Ministry of Education, General Directorate of Salah Alden Province Education, Tikrit, \\ Iraq \\ ${ }^{3}$ Department of Mathematics, Faculty of Science, University of Lagos, Akoka, Lagos, \\ Nigeria
}

\begin{abstract}
The purpose of this paper is to define and study a new class of sets called microgeneralized closed set and define micro-generalized continuous function and micro-generalized irresolute function in micro topological spaces. Basic properties of micro-generalized closed sets and its characterizations are analyzed.
\end{abstract}

2020 Mathematics Subject Classifications: 54C10, 18F60

Key Words and Phrases: Generalized closed, nano topology, nano-generalized closed, micro topology, micro-generalized closed, micro-generalized continuous function, micro-generalized irresolute functions

\section{Introduction}

The concept of closed set plays a fundamental role in General topology and real analysis. In 1970, Levine [11] introduced generalized closed sets in topological spaces. The notion of nano topology is introduced by Thivagar and Richard [13] whose idea of nano topological structure is grounded on lower, upper and boundary approximations of a subset of a universe set with an equivalence relation on it. They went further in the same reference to introduce the definition of closed, interior and closure set via concept of nano topology. In 2017, Bhuvaneswari [8] introduced the notion of nano topology and T. M. Al-shami [3] introduced Somewhere dense sets and ST1-spaces. The significant of nano topological structure is that it is needed to sort approximation to fit mathematical models of real-life problems. Azzam [7] expanded the nano topological structure by inserting grill

* Corresponding author.

DOI: https://doi.org/10.29020/nybg.ejpam.v14i4.3823

Email addresses: tahahameed@tu.edu.iq (T. H. Jasim),

saja.s.mohsen35504@st.tu.edu.iq (S. S. Mohsen), skanayo@unilag.edu.ng (K. S. Eke) 
changes to the lower approximation, upper approximation and boundary regions that resulted in grill nano topological spaces. The author equally defined grill nano-generalized closed sets which is an expansion of nano-generalized closed sets in grill nano topological spaces. In 2018, T. M. Al-shami [4] given that supra semi-compactness via supra topological spaces. In 2019, Chandrasekar [9] introduced the concept of micro topology which is simply an extension of nano topology and T. M. Al-shami and T. Noiri [6] provided more notions and mappings via somewhere dense sets. In 2020, Rasheed and Jasim [12] introduced micro- $\alpha$-open sets, micro- $\alpha$-continuous functions and some of their properties are investigated and T.M. Al-shami [10], [2], [1] and [5] studied respectively, Some applications of supra preopen sets, limit points and separation axioms with respect to supra semi-open sets, Para compactness on supra topological spaces and sum of the spaces on ordered setting. This research focus on the introduction of a new class of sets called microgeneralized closed set and micro-generalized continuous functions. Many characterizations and theorems relating to these sets are proved.

\section{Preliminaries}

In this section, we recall some preliminary definitions that led to the development of our main results.

\subsection{Definition [11]}

A subset $B$ from a topology $\tau$ on the space $X$ is said to be generalized closed set (shortly g-closed) if $C l .(B) \subset U$ for $B \subset U$ and $U$ is open in $(X, \tau)$. A set $B$ of topological space $(X, \tau)$ is called g-open if $X-B$ is g-closed.

\subsection{Definition $[9]$}

Let $X$ be a non-empty finite set of objects called 'universe' and let $R$ be an 'equivalence relation' on $X$ named as 'the indiscernibility relation'. Then $X$ divided into disjoint equivalence classes. Elements belonging to the same equivalence class are said to be indiscernible with one another. The pair $(X, R)$ is said to be 'the approximation space'. Let $A \subseteq X$,

(i) The lower approximation of $A$ with respect to $R$ is the set of all objects, which can be for certain classified as $A$ with respect to $R$ and it is denoted by $L R(A)$. That is $L R(A)=\cup(x \in X)\{R(x): R(x) \subseteq A$ where $R(x)\}$ denotes the equivalence class determined by $x \in X$.

(ii) The upper approximation of $A$ with respect to $R$ is the set of all objects, which can be possibly classified as $A$ with respect to $R$ and it is denoted by $U R(A)$. That is, $U R(A)=\cup(x \in X)\{R(x): R(x) \cap A \neq \phi\}$. 
(iii) The boundary region of $A$ with respect to $R$ is the set of all objects, which can be classified neither as $A$ nor as not- $A$ with respect to $R$ and it is denoted by $B R(A)$. That is, $B R(A)=U R(A)-L R(A)$.

\subsection{Definition [13]}

Let $X$ be the universe, $R$ be an equivalence relation on $X$ and $\tau_{R}(A)=\{\phi, X, L R(A)$, $U R(A), B R(A)\}$ where $A \subseteq X$. Then $\tau_{R}(A)$ satisfies the following axioms.

- $X$ and $\phi \in \tau_{R}(A)$.

- The union of the elements of any sub collection of $\tau R(A)$ is in $\tau_{R}(A)$.

- The intersection of the elements of any finite sub collection of $\tau_{R}(A)$ is in $\tau_{R}(A)$.

That is $\tau_{R}(A)$ forms a topology on $X$ called the Nano topology on $X$ with respect to $A$. We call $(X, \tau R(A))$ the Nano topological space.

The elements of $\tau_{R}(A)$ are called Nano open sets.

\subsection{Definition [13]}

If $\left(X, \tau_{R}(A)\right)$ is Nano topological space with respect to $A$ where $A \subseteq X$, if $B \subseteq X$, then

(i) The Nano interior of the set $B$ defined as the union of all Nano open subsets contained in $B$ is defined by N.Int.(B). That is N.Int.(B) is the greatest Nano open subset of $B$.

(ii) The Nano closure of the set $B$ defined as the intersection of all Nano closed containing $B$ is denoted by $N . C l .(B)$. That is $N . C l .(B)$ is the smallest Nano closed set containing $B$.

\subsection{Definition $[7]$}

A subset $B$ of $\left(X, \tau_{R}(A)\right)$ is called Nano-generalized closed sets (shortly N.g-closed) if $N . C l .(B) \subseteq U$ for $B \subseteq U$ and $U$ is Nano open in $(X, \tau R(A))$. A set $B$ of a Nano topological space $\left(X, \tau_{R}(A)\right)$ is called N.g-open if $X-B$ is N.g-closed.

\section{Micro-generalized closed set in Micro Topological Spaces}

In this section, the definition of Micro-generalized closed sets is introduced and some of its properties with related theorems are proved.

\subsection{Definition [9]}

If $\left(X, \tau_{R}(A)\right)$ is a Nano topological space then $\left.\mu_{(} R\right)(A)=N \cup\left(N^{\prime} \cap \mu\right): N, N^{\prime} \in \tau_{R}(A)$ is called Micro topology of $\tau_{R}(A)$ by $\mu$ where $\mu \notin \tau_{R}(A)$. 


\subsection{Definition [9]}

The Micro topology $\mu_{R}(A)$ satisfies the following axioms

(i) $X$ and $\emptyset \in \mu_{R}(A)$.

(ii) The union of the elements of any sub- collection form $\mu_{R}(A)$ is in $\mu_{R}(A)$.

(iii) The intersection of the elements of any finite sub collection of $\mu_{R}(A)$ is in $\mu_{R}(A)$.

Then $\mu_{R}(A)$ is called Micro topology on $X$ with respect to $A$. The triplet $\left(X, \tau_{R}(A), \mu_{R}(A)\right)$ is called Micro topological spaces and the elements of $\mu_{R}(A)$ are called Micro open sets and the complement of a Micro open set is called a Micro closed set.

\subsection{Definition [12]}

The Micro closure of a set $B$ is defined by Mic.Cl. $(B)=\cap\{F:$ FisMicroclosedset, $B \subseteq$ $F\}$ and the Micro interior of a set $B$ is defined by Mic.Int. $(B)=\cup\{U$ :UisMicroopenset, $U \subseteq$ $B\}$.

\subsection{Definition}

A sub set $\mathrm{B}$ of $\left(X, \tau_{R}(A), \mu_{R}(A)\right)$ is called Micro-generalized closed set ( shortly ,

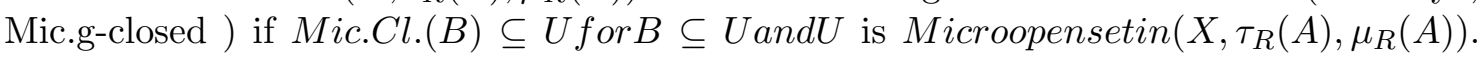
$\mathrm{A}$ set $\mathrm{B}$ of a Micro topological space $\left(X, \tau_{R}(A), \mu_{R}(A)\right)$ is called Mic.g-open if $\mathrm{X} \mathrm{B}$ is Mic.g-closed.

\subsection{Remark}

Every Micro closed set is Micro-generalized closed set.

\subsection{Example}

Let $X=\{1,2,3,4\}$ with $X / R=\{\{1\},\{3\},\{2,4\}\}$ and $A=\{1,2\}$. Then the Nano topology $\tau_{R}(A)=\{\emptyset, X,\{1\},\{2,4\},\{1,2,4\}\}$ which are Nano open sets, and the Nano closed sets $=\{\emptyset, X,\{3\},\{1\},,\{2,3,4\}\}$. Let $\mu=\{3\}$ then the Micro topology $\mu_{R}(A)=\{\emptyset, X,\{1\},\{3\},\{1,3\},\{2,4\},\{2,3,4\},\{1,2,4\}\}$ and the Micro closed sets $=$ $\{\emptyset, X,\{1\},\{3\},\{2,4\},\{1,3\},\{2,3,4\},\{1,2,4\}\}$. Show that the set $B=\{1,2,3\} \subseteq X$ is Mic.g-closed but not Micro-closed set.

\subsection{Proposition}

Every Nano open set is Micro open set.

Proof: Let $B \in \tau_{R}(A)$ and $\mu \notin \tau_{R}(A)$, Now from the definition of micro topology we have $B \cup(\emptyset \cap \mu)=B$ thus $B \in \mu_{R}(A)$. Hence $B$ is micro open set. 


\subsection{Remark}

The Nano topology and Micro topology are equivalent when $\mu \in \tau_{R}(A)$ and this clear from the definition of Micro topology (3.1).

\subsection{Theorem}

Every Nano-generalized closed set is Micro-generalized closed set. Proof: Suppose that $B$ is a Ng-closed set in $\left(X, \tau_{R}(A)\right)$ then Mic.Cl.(B) $\subseteq U$, for $B \subseteq U$ for all $U$ is Nano open set. Since every Nano open set is Micro open set (see proposition (3.7)). Then we get, $M i c . C l(B) \subseteq U$, for $B \subseteq U$ for all $U$ is Micro open set. Thus, the set $B$ is Mic.g-closed set.

\subsection{Example}

In the same example (3.6) above let $B=\{1,2\} \subseteq\{1,2,4\} \in \tau_{R}(A)$ and N.Cl. $\{1,2\}=$ $X \nsubseteq\{1,2,4\}$.Then $B$ is not Nano-generalized closed set .But $B=\{1,2\} \subseteq\{1,2,4\} \in$ $\mu_{R}(A)$ such that Mic.Cl. $\{1,2\}=\{1,2,4\}$ thus $B$ is Micro-generalized closed set.

\subsection{Theorem}

Let $\left(X, \tau_{R}(A), \mu_{R}(A)\right)$ be a Micro topological space. If $B$ and $C$ are Micro-generalized closed sets, then $B \cup C$ is Micro-generalized closed set.

Proof : Let $B$ and $C$ are Mic.g-closed sets. Then Mic.Cl.(B) $\subseteq U$ when every $B \subseteq U$ and $U$ is Micro open set and Mic.Cl. $(C) \subseteq V$ for $C \subseteq V$ and $V$ is Micro open set. Since $B$ is subset of $U$ and $C$ is subset of $V$, thus $B \cup C \subseteq U \cup V$ and $U \cup V$ is Micro open set.

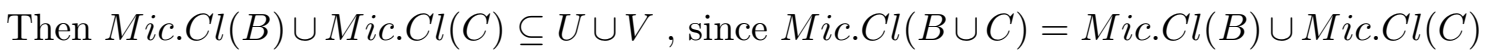
then Mic.Cl( $B \cup C) \subseteq U \cup V$ for $B \cup C \subseteq U \cup V$ and $U \cup V$ is Micro open set. which leads to $B \cup C$ is Micro-generalized closed set.

\subsection{Proposition}

Let $\left(X, \tau_{R}(A), \mu_{R}(A)\right)$ be a Micro topological space and $B a n d C$ are subset of $X$ such that $B \subseteq C$, then :

(i) If $\mathrm{B}$ is a Micro-generalized closed set then it is not necessary that $C$ is Microgeneralized closed set.

(ii) If $C$ is a Micro-generalized closed set then it is not necessary that $B$ is Microgeneralized closed set.

The proposition above needs the following example: 


\subsection{Example}

Let $X=\{a, b, c, d\}$ with $X / R=\{\{a\},\{c\},\{b, d\}\}$ and $A=\{b, d\}$. Then $\tau_{R}(A)=$ $\{\emptyset, X,\{b, d\}\}$. Let $\mu=\{a, b, c\}$, Then $\mu_{R}(A)=\{\emptyset, X,\{b\},\{b, d\},\{a, b, c\}\}$. We have :

(i) The set $B=\{a, c\}$ is Mic.g-closed but the set $C=\{a, b, c\}$ is not Mic.g-closed.

(ii) The set $C=\{b, c, d\}$ is Mic.g-closed but the set $B=\{b, c\}$ is not Mic.g-closed.

\subsection{Remark}

Let $\left(X, \tau_{R}(A), \mu_{R}(A)\right)$ be a Micro topological space. If $B$ and $C$ are Micro-generalized closed sets, then $B \cap C$ need not be a Micro-generalized closed set, we can consider the set $B=\{a, b, d\}$ and the set $C=\{b, c, d\}$ in the example (3.13) each of them are Microgeneralized closed set but $B \cap C=\{b, d\}$ is not Micro-generalized closed set.

\subsection{Proposition}

Let $\left(X, \tau_{R}(A), \mu_{R}(A)\right)$ be a Micro topological space. If $B$ is not Micro open set and not subset of any Micro open set in $X$ (just $B \subseteq X$ ) then $B$ is Micro-generalized closed set.

Proof: Let $B \notin \mu_{R}(A)$ and not subset of any Micro open set in $X$ (just $B \subseteq X$ ). Then $\operatorname{Mic.} C l(B) \subseteq C$ where $C$ is Micro closed set and $C \subseteq X$. Thus $\operatorname{Mic.} C l(B) \subseteq X$. That is, $B$ is Mic.g-closed set in $X$.

\subsection{Example}

In the example (3.13), Let $\mu=\{a\}$ Then $\mu_{R}(A)=\{\emptyset, X,\{a\},\{b, d\},\{a, b, d\}\}$. The set $B=\{c\}$ is not Micro open set and not subset of any Micro open set in $X$ (just $B \subseteq X$ ). Then $\operatorname{Mic.Cl}(B)=\{c\} \subseteq X$ where $X$ is Micro open set, So $B$ is Mic.g-closed set in $X$.

\subsection{Remark}

If every set in a micro topology is micro open, then a micro topology is the discrete. Hence, every set is both micro open and micro g-closed.

\section{Micro-generalized continuous function and micro-generalized irresolute function}

In this segment we present the definition of Micro-generalized continuous function and the micro-generalized irresolute function. 


\subsection{Definition}

Let $\left(X, \tau_{R}(A), \mu_{R}(A)\right)$ and $\left(Y, \tau_{R}^{\prime}(A), \mu_{R}^{\prime}(A)\right)$ be two Micro topological spaces. A function $f: X \rightarrow Y$ is called Micro-generalized continuous function (shortly Mic.g-continuous) if $f^{-1}(B)$ is Mic.g-closed set in $\mathrm{X}$ for every Micro-closed set BinY.

\subsection{Example}

Let $X=\{1,2,3,4,5\}$, with $X / R=\{\{1\},\{5\},\{2,3,4\}\}$ and $A=\{1,2\} \subseteq X$. Then $\tau_{R}(A)=\{\emptyset, X,\{1\},\{2,3,4\},\{1,2,3,4\}\}$. Let $\mu=\{2\}$, Then

$\mu_{R}(A)=\{\emptyset, X,\{1\},\{2\},\{1,2\},\{2,3,4\},\{1,2,3,4\}\}$ and

the Micro closed sets $=\{\emptyset, X,\{5\},\{1,5\},\{3,4,5\}\{1,3,4,5\},\{2,3,4,5\}\}$. Let $Y=\{p, q, r, s, t\}$, with $Y / R=\{\{s\},\{t\},\{p, q, r\}\}$ and $A=\{p, q\} \subseteq Y$. Then $\tau_{R}^{\prime}(A)=\{\emptyset, Y,\{p, q, r\}\}$. Let $\tau^{\prime}=\{s\}$, Then $\mu_{R}^{\prime}(A)=\{\emptyset, Y,\{s\},\{p, q, r\},\{p, q, r, s\}\}$ and the Micro closed sets $=\{\emptyset, Y,\{t\},\{s, t\},\{p, q, r, t\}\}$.

Let $f: X \rightarrow Y$ be a function defined as $f(1)=s, f(2)=q, f(3)=r, f(4)=p, f(5)=t$. Micro closed set in $Y$ are $\{t\},\{s, t\},\{p, q, r, t\}$ and the sets $\{5\},\{\{1,5\},\{2,3,4,5\}$ are Mic.g-closed sets in $X$. thus $f^{-1}(B)$ is Mic.g-closed set in $X$ for every Micro-closed set $\operatorname{Bin} Y$. Then $f$ is Micro-generalized continuous function.

\subsection{Definition}

Let $\left(X, \tau_{R}(A), \mu_{R}(A)\right)$ and $\left(Y, \tau_{R}^{\prime}(A), \mu_{R}^{\prime}(A)\right)$ be two Micro topological spaces. A function $f: X \rightarrow Y$ is called micro-generalized irresolute function if $f^{-1}(B)$ is Mic.g-closed set in $X$ for every Micro.g-closed set Bin $Y$.

\subsection{Example}

Let $X=\{a, b, c\}$ with $X / R=\{\{a\},\{b, c\}\}$ and $A=\{b, c\} \subseteq X$. Then $\tau_{R}(A)=$ $\{\emptyset, X,\{b, c\}\}$. Let $\mu=\{a\}$, Then $\mu_{R}(A)=\{\emptyset, X,\{a\},\{b, c\}$. Let $Y=\{1,2,3\}$, with $Y / R=\{\{2\},\{1,3\}\}$ and $A=\{1,3\} \subseteq Y$, Then $\tau_{R}(A)=\{\emptyset, Y,\{1,3\}\}$. Let $\mu^{\prime}=\{2\}$, Then $\mu_{R}^{\prime}(A)=\{\emptyset, Y,\{2\},\{1,3\}\}$. Let $f: X \rightarrow Y$ be a function defined as $f(a)=$ $2, f(b)=1, f(c)=3$. Mic.g-closed sets in $X$ are $\{a\},\{b\},\{c\},\{a, b\},\{a, c\},\{b, c\}$ and Mic.g-closed sets in $Y$ are $\{1\},\{2\},\{3\},\{1,2\},\{1,3\},\{2,3\}$. Therefore for every Mic.gclosed set $\operatorname{Bin} Y, f^{-1}(B)$ is Mic.g-closed set in $X$. Then $f$ is micro-generalized irresolute function

\subsection{Theorem}

Let $f: X \rightarrow Y$ be a function from Micro Topological space $\left(X, \tau_{R}(A), \mu_{R}(A)\right)$ to Micro Topological space $\left(Y, \tau_{R}^{\prime}(A), \mu_{R}^{\prime}(A)\right)$. If $f: X \rightarrow Y$ is Micro continuous function [12], then $f: X \rightarrow Y$ is Micro-generalized continuous function.

Proof: suppose that the function $f: X \rightarrow Y$ is Micro continuous function from Micro Topological space $\left(X, \tau_{R}(A), \mu_{R}(A)\right)$ to Micro Topological space $\left(Y, \tau_{R}^{\prime}(A), \mu_{R}^{\prime}(A)\right)$. That is the inverse Image of any Micro-closed set in $\left(Y, \tau_{R}^{\prime}(A), \mu_{R}^{\prime}(A)\right)$ is Micro-closed set 
in $\left(X, \tau_{R}(A), \mu_{R}(A)\right)$. Let $B$ be a Micro-closed set in $\left(Y, \tau_{R}^{\prime}(A), \mu_{R}^{\prime}(A)\right)$. Then $f^{-1}(B)$ is Micro-closed set in $\left(X, \tau_{R}(A), \mu_{R}(A)\right)$. Now By remark 3.5 (Every Micro closed set is Micro-generalized closed set) we get, $f^{-1}(B)$ is Micro-generalized closed set in $\left(X, \tau_{R}(A), \mu_{R}(A)\right)$. Thus $f: X \rightarrow Y$ is Micro-generalized continuous function.

\subsection{Theorem}

Every micro-generalized irresolute function is Micro-generalized continuous function. Proof: Suppose that the function $f: X \rightarrow Y$ is micro-generalized irresolute function from Micro Topological space $\left(X, \tau_{R}(A), \mu_{R}(A)\right)$ to Micro Topological space $\left(Y, \tau_{R}^{\prime}(A), \mu_{R}^{\prime}(A)\right)$. We want to prove that $\mathrm{f}: X \rightarrow Y$ is Micro-generalized continuous function. Let $B$ be Microclosed set in $\left(Y, \tau_{R}^{\prime}(A), \mu_{R}^{\prime}(A)\right)$ then $f^{-1}(B)$ is Micro-closed set in $\left(X, \tau_{R}(A), \mu_{R}(A)\right)$ and by remark 3.5 (Every Micro closed set is Micro-generalized closed set) we get, $f^{-1}(B)$ is Micro-generalized closed set in $\left(X, \tau_{R}(A), \mu_{R}(A)\right)$. Thus $f: X \rightarrow Y$ is Micro-generalized continuous function.

\subsection{Definition}

Let $\left(X, \tau_{R}(A), \mu_{R}(A)\right)$ and $\left(Y, \tau_{R}^{\prime}(A), \mu_{R}^{\prime}(A)\right)$ be two Micro topological spaces. A function $f: X \rightarrow Y$ is called Micro-generalized continuous function at a point $c \in X$ if for every Micro-closed set $G$ containing $f(c) i n Y$, there are exist a Mic.g-closed $B$ containing $\operatorname{cin} X$ such that $f(B) \subseteq G$.

\subsection{Theorem}

Let $\left(X, \tau_{R}(A), \mu_{R}(A)\right)$ and $\left(Y, \tau_{R}^{\prime}(A), \mu_{R}^{\prime}(A)\right)$ be two Micro topological spaces. A function $f: X \rightarrow Y$ is Micro-generalized continuous function if and only if $f$ is Microgeneralized continuous function at each point of $X$.

Proof: Let $f: X \rightarrow Y$ be Mic.g-continuous. Let $c \in X$, and $G$ be a Micro-closed set in $Y$ containing $f(c)$. Since $\mathrm{f}$ is Mic.g-continuous, $f^{-1}(G)$ is Mic.g-closed in $X$ containing $c$. Let $B=f^{-1}(G)$, then $f(G) f(B) \subseteq G$, and $f(a) \in B$. Hence $f$ is continuous at $c$. Conversely, suppose $f$ is Mic.g-continuous at each point of $X$. Let $G$ be Micro-closed set in $Y$. If $f^{-1}(G)=\emptyset$ then it is Mic.g-closed. So let $f^{-1}(G) \neq \emptyset$. Take any $c \in f^{-1}(G)$, then $f(c) \in G$. Since $f$ is Mic.g-continuous at each point there exist a Mic.g-closed set $B_{c}$ containing $c$ such that $f\left(B_{c}\right) \subseteq G$. Let $B=\left(B_{c} \mid c \in f^{-1}(G)\right)$. Claim: $B=f^{-1}(G)$ If $a \in f^{-1}(G)$ then $a \in B_{a} \subseteq B$. Hence $f^{-1}(G) \subseteq B$. On the other hand, suppose $b \in B$ then $b \in B_{c}$ for some $c$ and $b \in f^{-1}(G)$. Hence $B=f^{-1}(G)$. Since $B_{c}$ is Mic.g-closed set, by dentition (4.7) $B$ is Mic.g-closed and hence $B=f^{-1}(G)$ is Mic.g-closed set for every Micro-closed set $G$ in $Y$. Hence $\mathrm{f}$ is Mic.g-continuous.

\subsection{Theorem}

Let $\left(X, \tau_{R}(A), \mu_{R}(A)\right)$ and $\left(Y, \tau_{R}^{\prime}(A), \mu_{R}^{\prime}(A)\right)$ be two Micro topological spaces. Then $f: X \rightarrow Y$ is Micro-generalized continuous function if and only if $f^{-1}(B)$ is Mic.g-open 
in $X$ whenever $B$ is Micro- open in $Y$.

Proof: Let $f: X \rightarrow Y$ is Micro-generalized continuous function and $B$ be Micro-open in $Y$. Then $B^{c}$ is Micro-closed in $Y$. By hypothesis $f^{-1}\left(B^{c}\right)$ is Mic.g-closed in $X$, i.e., $\left[f^{-1}(B)\right]^{c}$ is Mic.g-closed set in $X$. Hence $f^{-1}(B)$ is Mic.g-open in $X$. Whenever $B$ is Micro-open in $Y$. Conversely, suppose $f^{-1}(B)$ is Mic.g-open set in $X$ whenever $B$ is Micro-open in $Y$. Let $H$ is Micro-closed in $Y$ then $H^{c}$ is Micro-open in $Y$. By assumption $f^{-1}\left(H^{c}\right)$ is Mic.g-open in $X$.i.e., $\left[f^{-1}(H)\right]^{c}$ is Mic.g-open in $X$. Then $f^{-1}(H)$ is Mic.g-closed in $X$. Hence $f$ is Mic.g-continuous function.

\subsection{Theorem}

Let $\left(X, \tau_{R}(A), \mu_{R}(A)\right),\left(Y, \tau_{R}^{\prime}(A), \mu_{R}^{\prime}(A)\right)$ and $\left(Z, \tau_{R}^{\prime \prime}(A), \mu_{R}^{\prime \prime}(A)\right)$ be three Micro-topological spaces. If $f: X \rightarrow Y$ and $g: Y \rightarrow Z$ are micro-generalized irresolute functions then $g \circ f: X \rightarrow Z$ is also micro-generalized irresolute function.

Proof: Let $B$ be a Mic.g-closed set in $Z$. Since by $g$ is micro-generalized irresolute function, then $g^{-1}(B)$ is Mic.g-closed set in $Y$. Now since by $f$ is micro-generalized irresolute function, then $f^{-1}\left(g^{-1}(B)\right)$ is Mic.g-closed set in $X$ but $(g \circ f)^{-1}(B)=\left(f^{-1} \circ g^{-1}\right)(B)=$ $f^{-1}\left(g^{-1}(B)\right)$. Thus $(g \circ f)^{-1}(B)$ is Mic.g-closed set in $X$. Hence $g \circ f$ is micro-generalized irresolute function.

\subsection{Theorem}

Let $\left(X, \tau_{R}(A), \mu_{R}(A)\right),\left(Y, \tau_{R}^{\prime}(A), \mu_{R}^{\prime}(A)\right)$ and $\left(Z, \tau_{R}^{\prime \prime}(A), \mu_{R}^{\prime \prime}(A)\right)$ be three Micro-topological spaces. If $f: X \rightarrow Y$ is a micro-generalized continuous function and $g: Y \rightarrow Z$ be a Micro-continuous function then $g \circ f: X \rightarrow Z$ is micro-generalized continuous function. Proof: Let $B$ be a Micro-closed set in $Z$. Since by $g$ is Micro-continuous function, then $g^{-1}(B)$ is Micro-closed set in $Y$. Now since by $f$ is micro-generalized continuous function, then $f^{-1}\left(g^{-1}(B)\right)$ is micro-generalized closed set in $X$ but $(g \circ f)^{-1}(B)=\left(f^{-1} \circ g^{-1}\right)(B)=$ $f^{-1}\left(g^{-1}(B)\right)$. Thus $(g \circ f)^{-1}(B)$ is micro-generalized closed set in $X$. Hence $g \circ f$ is microgeneralized continuous function.

\section{References}

[1] T Al-shami. Paracompactness on supra topological spaces. Journal of Linear and Topological Algebra (JLTA), 9(02):121-127, 2020.

[2] Tareq M AL-shami, EA Abo-Tabl, Baravan Assad, and Mohamed Arahet. Limit points and separation axioms with respect to supra semi-open sets. European Journal of Pure and Applied Mathematics, 13(3):427-443, 2020.

[3] TM Al-Shami. Somewhere dense sets and st1-spaces. Punjab University Journal of Mathematics, 49(2):101-111, 2017.

[4] TM Al-Shami. Supra semi-compactness via supra topological spaces. Journal of Taibah University for Science, 12(3):338-343, 2018. 
[5] TM Al-shami. Sum of the spaces on ordered setting. Moroccan J. of Pure and Appl. Anal, 6(2):255-265, 2020.

[6] TM Al-Shami and T Noiri. More notions and mappings via somewhere dense sets. Afrika Matematika, 30(7):1011-1024, 2019.

[7] AA Azzam. Grill nano topological spaces with grill nano generalized closed sets. Journal of the Egyptian Mathematical Society, 25(2):164-166, 2017.

[8] M Bhuvaneswari. A study on nano topology. A Journal of Nehru Arts and Science College, 5(1), 2017.

[9] Sakkraiveeranan Chandrasekar. On micro topological spaces. Journal of New Theory, (26):23-31, 2019.

[10] ME El-Shafei, AH Zakari, and TM Al-Shami. Some applications of supra preopen sets. Journal of Mathematics, 2020, 2020.

[11] Norman Levine. Generalized closed sets in topology. Rendiconti del Circolo Matematico di Palermo, 19(1):89-96, 1970.

[12] Reem O Rasheed and Taha H Jasim. On micro- $\alpha$-open sets and micro- $\alpha$-continuous functions in micro topological spaces. In Journal of Physics: Conference Series, volume 1530, page 012061. IOP Publishing, 2020.

[13] M Lellis Thivagar and Carmel Richard. On nano forms of weakly open sets. International journal of mathematics and statistics invention, 1(1):31-37, 2013. 